\title{
Adrenal incidentalomas: a simple guide to a disease of modern technology
}

\author{
${ }^{1} \mathrm{~S}$ Ghosh, ${ }^{2} \mathrm{M}$ Jones, ${ }^{3} \mathrm{~K}$ Swaminathan \\ ${ }^{1}$ Specialist Registrar in Acute Medicine; ${ }^{2}$ Consultant Radiologist; ${ }^{3}$ Consultant Endocrinologist, Victoria Hospital, Kirkcaldy, UK
}

ABSTRACT Incidentally discovered adrenal masses (adrenal incidentalomas) found during radiological procedures are a by-product of modern-day medicine. Incidental findings of such adrenal masses pose uncertainties and dilemmas in evaluation and management. There is a paucity of comprehensive guidelines from professional societies, and current recommendations (based on expert opinions and the US National Institutes of Health statement, 2002) are open to debate with regards to cost and clinical benefit. We report two patients with 'adrenal incidentalomas' with different outcomes during the course of evaluation. We conclude with a simple guide to the evaluation and management of such masses based on the available literature.
Correspondence to K Swaminathan, Victoria Hospital, Kirkcaldy KY2 5AH, UK

tel. +44 (0) 1592643355 ext. 1582 e-mail

krishnan.swaminathan@nhs.net

KEYWORDS Adrenal incidentaloma, Cushing's syndrome, phaeochromocytoma

DECLARATION OF INTERESTS No conflict of interests declared.

\section{CASE REPORT}

Case I

A 33-year-old woman was found to have an incidental adrenal mass during ultrasound imaging for recurrent urinary tract infections. This was subsequently confirmed by computed tomography (CT), which showed a wellcircumscribed $3 \times 3.7 \mathrm{~cm}$ lesion with a density of 40 Hounsfield units (HU) (Figure I). She was clinically Cushingoid with a history of hypertension, impaired glucose tolerance, hirsutism and amenorrhoea. Adrenocorticotrophic hormone (ACTH) independent Cushing's syndrome was confirmed based on the elevated urine 24-hour cortisol/creatinine ratio (269 $\mathrm{nmol} / \mathrm{mmol}$, normal range $0-25)$, undetectable levels of serum ACTH (<I milliunits/litre, normal range 2-20) and lack of cortisol suppression with dexamethasone administration (serum cortisol of $56 \mathrm{I} \mathrm{nmol/l} \mathrm{after} \mathrm{two}$ days of $0.5 \mathrm{mg}$ dexamethasone). She had a laparoscopic adrenalectomy with good effect.

\section{Case 2}

A 62-year-old man had a large irregular solid-cystic 'adrenal incidentaloma' during routine imaging after a recently diagnosed prostatic carcinoma (Figure 2). History and clinical examination was non-contributory except for a blood pressure of 170/100. Urine 24-hour collections showed a nearly fourfold elevation of normetadrenaline ( $2.9 \mu \mathrm{mol}$ )/collection, normal range 0.4-3.4 $\mathrm{\mu mol} /$ collection). Random aldosterone-renin ratio and a $\mathrm{I} \mathrm{mg}$ dexamethasone suppression test were within normal limits. Careful pre-operative alpha and beta blockade was followed by a successful resection of a large phaeochromocytoma. Histopathology showed cells expressing chromogranin A and synaptophysin consistent with a phaeochromocytoma. Urine 24-hour collections for fractionated metanephrines were within normal limits postoperatively. The patient is under regular follow-up.

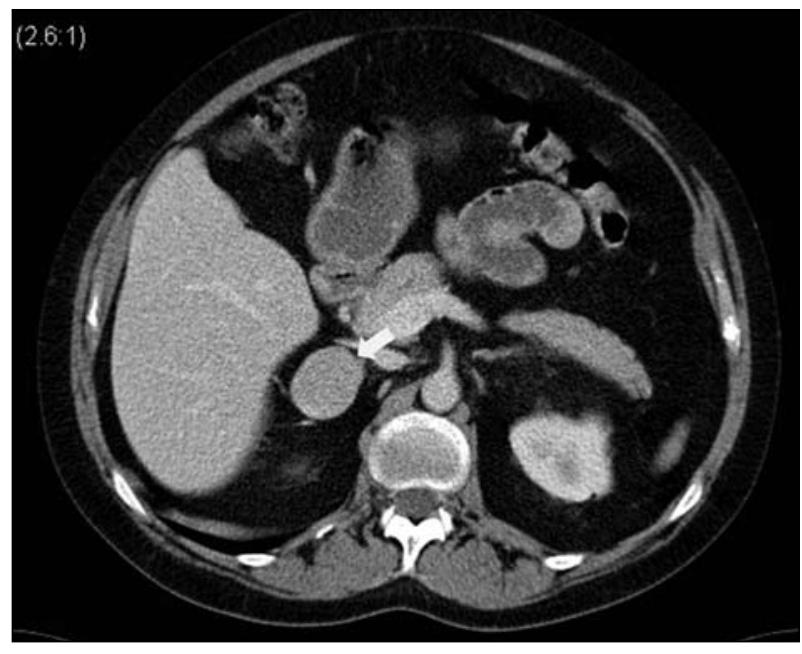

FIGURE I Computed tomography showing a $3 \times 3.7 \mathrm{~cm}$ right adrenal adenoma with a density of $40 \mathrm{HU}$ in a female patient suffering from recurrent urinary tract infections.

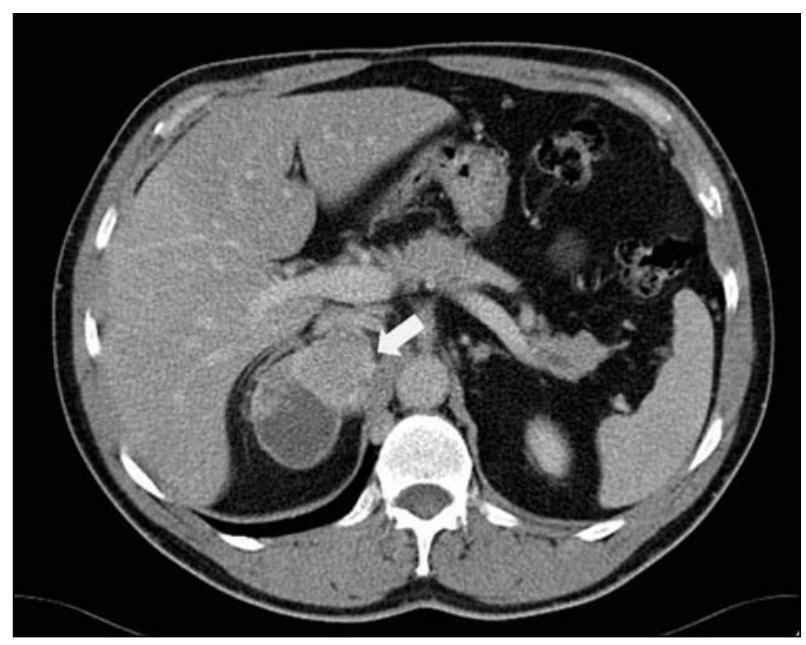

FIGURE 2 A male patient was found to have a $7.6 \times 4.7 \mathrm{~cm}$ solid-cystic right adrenal lesion during routine imaging. 


\section{DISCUSSION}

Adrenal incidentalomas are defined as a 'serendipitous discovery of an adrenal mass, $\mathrm{I} \mathrm{cm}$ or more in diameter during a radiological investigation performed for indications other than an evaluation of adrenal disease'.' Adrenal incidentalomas are increasingly recognised in current practice, ${ }^{2,3}$ due to the routine use of sensitive and sophisticated imaging techniques, especially CT imaging, with a reported prevalence of $4.4 \% .{ }^{4}$ Adrenal incidentalomas pose a challenge to physicians and surgeons as there are no comprehensive guidelines from professional societies. In this article, we highlight two interesting cases of 'adrenal incidentalomas', which turned out to be functioning tumours that could have caused potential cardiovascular sequelae in the long term if not appropriately evaluated.

While the optimal strategy to evaluate an adrenal incidentaloma is not clear, the two questions that need to be asked by the physician are a) whether the mass is malignant and b) whether it is functioning.

The two major predictors of malignancy in an adrenal mass are the size of the mass and image characteristics. In a study of 887 patients with adrenal incidentalomas, $90 \%$ of adrenocortical carcinomas were $>4 \mathrm{~cm}$ in diameter when discovered. ${ }^{5}$ But size alone is not an absolute predictor of malignancy, as in the same study the specificity was quite low, with $76 \%$ of incidentalomas $>4 \mathrm{~cm}$ reported as benign. Hence, it is vital to review the imaging characteristics to improve the predictive value of diagnosis. The most useful parameter in the radiologist's report would be the density of image in HU on a noncontrast CT scan. If an adrenal incidentaloma is reported as $<10 \mathrm{HU}$ on a non-contrast scan, the likelihood of a benign adenoma is virtually certain. ${ }^{6}$ When delayed contrast-enhanced CT is performed, most benign adenomas exhibit a rapid washout of contrast ( $>50 \%$ washout in 10 minutes) compared to other neoplasms (for example, phaeochromocytomas, primary malignancy and metastases). In fact, this feature was reported to be $100 \%$ sensitive and specific for a benign adenoma, at least in one study. ${ }^{7}$ Other imaging characteristics that are highly suggestive of malignancy are irregular borders, inhomogeneous densities and tumour calcification.

In terms of functionality, approximately 15\% of adrenal incidentalomas are functional. ${ }^{8}$ Endocrine evaluation should be performed in all patients with adrenal incidentalomas to identify silent states of hormonal excess. A focused history and a thorough clinical examination can be rewarding. All patients should have a I mg dexamethasone suppression test and urine 24-hour collection for fractionated metanephrines, irrespective of the presence or absence of clinical features of Cushing's syndrome or phaeochromocytomas.

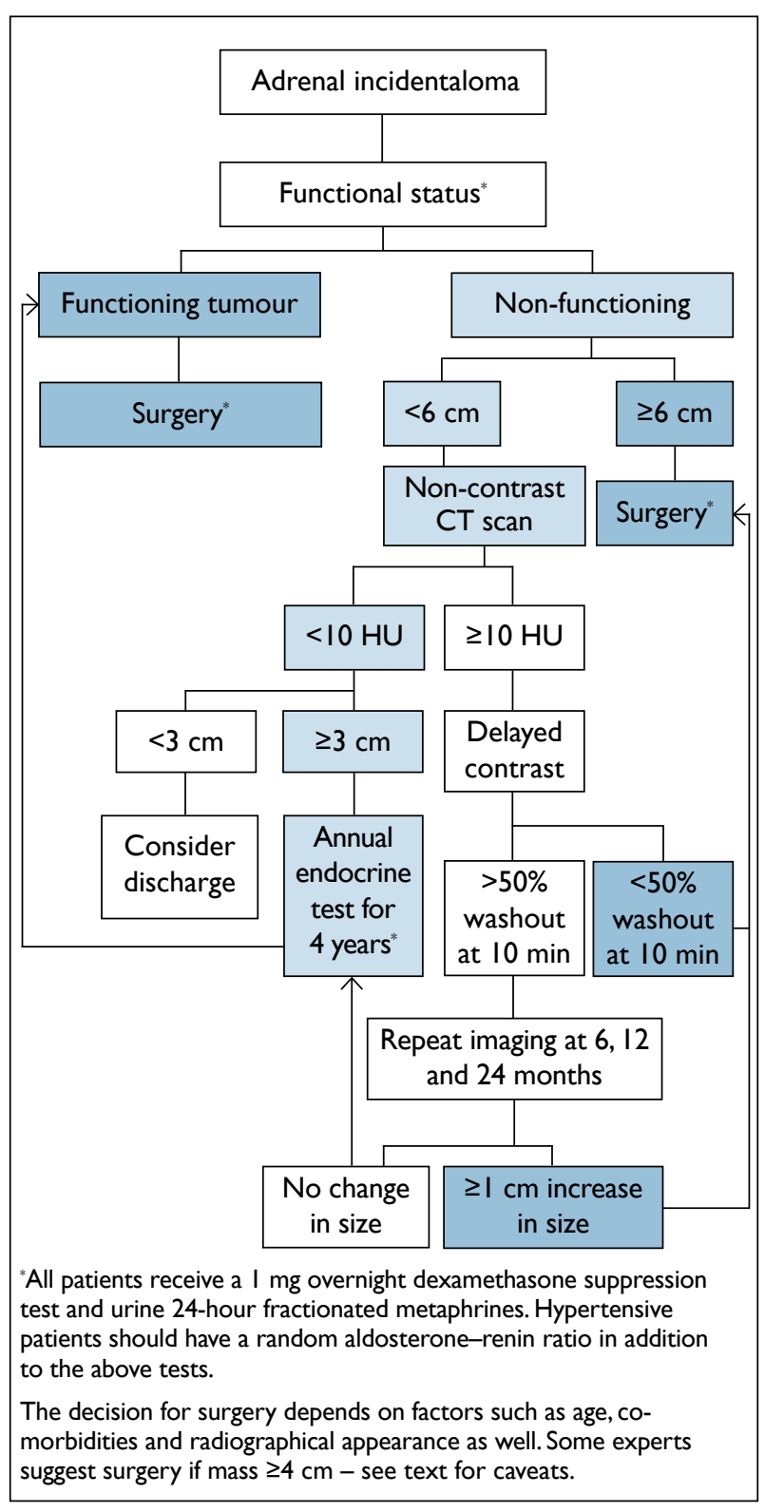

FIGURE 3 Adrenal incidentaloma flow chart.

Subclinical Cushing's syndrome (mild hypercortisolism with no obvious stigmata of Cushing's syndrome) is the most common hormonal abnormality in patients with adrenal incidentalomas. ' A cortisol cut-off of $138 \mathrm{nmol} / \mathrm{l}$ after an overnight dose of $\mathrm{I} \mathrm{mg}$ dexamethasone is thought to reflect cortisol secretory autonomy..$^{10}$ In such patients, further confirmatory tests should be done to exclude a false positive test result. Phaeochromocytomas are thought to account for about 5\%' of adrenal incidentalomas. These tumours may be clinically silent and potentially lethal if missed, as in Case 2. In patients with hypertension, in addition to the above tests, a random plasma aldosterone and renin activity should be measured to evaluate the possibility of Conn's syndrome. ${ }^{6}$

All patients with adrenal incidentaloma and biochemical evidence of phaeochromocytoma, Conn's syndrome and Cushing's syndrome should be considered for surgery. 
Subclinical Cushing's, both as an entity and a referral criteria for surgery, is highly debatable and controversial. In the absence of evidence from randomised controlled trials, some experts advocate surgical referral for young people below the age of 40 years or patients having other markers of autonomous cortisol secretion such as diabetes, osteoporosis or worsening of hypertension."

For those with non-functioning tumours, the decision to operate is based on the size of the tumour, imaging characteristics, growth rate and co-morbidities (Figure 3). ${ }^{6,11,12}$ There are a few caveats to remember in this flow chart. The yield and cost-effectiveness of repeat imaging at six, 12 and 24 months is not clear" as prospective data are sparse. If the imaging characteristic is highly suspicious, repeat imaging at three months is often recommended." The yield and cost-effectiveness of annual endocrine testing in non-functioning tumours at baseline is not known either, and this recommendation is based on a few studies that have shown hormonal hypersecretion later, ${ }^{13-15}$ especially if the mass is $>3 \mathrm{~cm} .{ }^{15}$
The risk of hormonal hyperfunction in an adrenal incidentaloma with a low attenuation value and $<3 \mathrm{~cm}$ is extremely low; the decision to discharge such patients is debatable. Finally, some experts advocate surgery if the mass is $\geq 4 \mathrm{~cm}$," but the decision to operate depends on other confounding factors such as age, co-morbidities and imaging characteristics, since surgery may be clearly inappropriate in certain circumstances; it may be a better option in younger patients.

\section{CONCLUSION}

Adrenal incidentalomas are increasingly identified due to advances in imaging techniques. The optimal strategy to evaluate such masses is not entirely clear. The density of the mass in $\mathrm{HU}$ in a non-contrast CT scan is helpful, and all adrenal incidentalomas should be assessed for functionality. Any adrenal mass $>6 \mathrm{~cm}$ and any functional adrenal mass should be considered for surgery. Noncontrast CT will be helpful to decide treatment options in adrenal incidentalomas $<6 \mathrm{~cm}$.

\section{REFERENCES}

I Young WF. Management approaches to adrenal incidentalomas: a view from Rochester, Minnesota. Endocrinol Metab Clin North Am 2000; 29:I59-85. doi:I0.10I6/S0889-8529(05)70I22-5

2 Kloos RT, Gross MD, Francis IR et al. Incidentally discovered adrenal masses. Endocr Rev 1995; 16:460-84.

3 Aron DC. The adrenal incidentaloma: disease of modern technology and public health problem. Rev Endocr Metabol Disord 200I; 2:335-42. doi:I0.I023/A:I0II5808I9I32

4 Bovio S, Cataldi A, Reimondo $G$ et al. Prevalence of adrenal incidentaloma in a contemporary computerized tomography series. J Endocrinol Invest 2006; 29:298-302.

5 Angeli A, Osella G, Ali A et al. Adrenal incidentaloma: an overview of clinical and epidemiological data from the National Italian Study Group. Horm Res 1997; 47:279-83. doi:I0.II59/000I85477

6 Grumbach MM, Biller BM, Braunstein GD et al. Management of the clinically inapparent adrenal mass ('incidentaloma'). Ann Intern Med 2003; 138:424-9.

7 Szolar DH, Korobkin M, Reittner $\mathrm{P}$ et al. Adrenocortical carcinomas and adrenal pheochromocytomas: mass and enhancement loss evaluation at delayed contrast-enhanced CT. Radiology 2005; 234:479-85. doi:I0.I |48/radiol.234203 I876

8 Mantero F, Terzolo M, Arnaldi G et al. A survey on adrenal incidentaloma in Italy. Study Group on Adrenal Tumors of the Italian Society of Endocrinology. J Clin Endocrinol Metab 2000; 85:637-44. doi:10.1210/jc.85.2.637

9 Reincke M. Subclinical Cushing's syndrome. Endocrinol Metab Clin North Am 2000; 29:43-56. doi: I 0. I0 I6/S0889-8529(05)70I I5-8

10 Tsagarakis S, Vassiliadi D, Thalassinos N. Endogenous subclinical hypercortisolism: diagnostic uncertainties and clinical implications. J Endocrinol Invest 2006; 29:47I-82.

I I Young WF.The incidentally discovered adrenal mass. N Engl J Med 2007; 356:60I-10. doi:I0.1056/NEJMcp065470

12 Gopan T, Remer E, Hamrahian A. Evaluating and managing adrenal incidentalomas. Cleve Clin J Med 2006; 73:56I-8. doi:I0.3949/ ccjm.73.6.56I

13 Libe R, Dall'Asta C, Barbetta $L$ et al. Long-term follow-up study of patients with adrenal incidentalomas. Eur J Endocrinol 2002; 147:489-94. doi:10.1530/eje.0.1470489

I4 Bulow B, Jansson S, Juhlin C et al. Adrenal incidentaloma - followup results from a Swedish prospective study. Eur J Endocrinol 2006; 154:419-23. doi:10.1530/eje.1.02110

15 Barzon L, Scaroni C, Sonino $\mathrm{N}$ et al. Risk factors and long-term follow-up of adrenal incidentalomas. J Clin Endocrinol Metab 1999; 84:520-6. doi:10.1210/jc.84.2.520 